\title{
Towards quantitative PET/MRI: a review of MR-based attenuation correction techniques
}

\author{
Matthias Hofmann • Bernd Pichler • \\ Bernhard Schölkopf • Thomas Beyer
}

Published online: 23 December 2008

(C) The Author(s) 2008. This article is published with open access at Springerlink.com

\begin{abstract}
Introduction Positron emission tomography (PET) is a fully quantitative technology for imaging metabolic pathways and dynamic processes in vivo. Attenuation correction of raw PET data is a prerequisite for quantification and is typically based on separate transmission measurements. In $\mathrm{PET} / \mathrm{CT}$ attenuation correction, however, is performed routinely based on the available CT transmission data. Objective Recently, combined PET/magnetic resonance (MR) has been proposed as a viable alternative to PET/
\end{abstract}

M. Hofmann $(\bowtie) \cdot$ B. Schölkopf

Max Planck Institute for Biological Cybernetics,

Spemannstraße 38,

72076 Tuebingen, Germany

e-mail: matthias.hofmann@tuebingen.mpg.de

M. Hofmann • B. Pichler

Laboratory for Preclinical Imaging and Imaging Technology

of the Werner Siemens-Foundation, Department of Radiology,

University of Tuebingen,

Roentgenweg 11,

72076 Tuebingen, Germany

\section{Hofmann}

Wolfson Medical Vision Laboratory,

Department of Engineering Science, University of Oxford,

Oxford OX1 3PJ, UK

\section{T. Beyer}

Department of Nuclear Medicine, University Hospital Duisburg-Essen,

Hufelandstr 55,

45122 Essen, Germany

T. Beyer

cmi-experts $\mathrm{GmbH}$,

Pestalozzistr,

8032 Zurich, Switzerland
CT. Current concepts of PET/MRI do not include CT-like transmission sources and, therefore, alternative methods of PET attenuation correction must be found. This article reviews existing approaches to MR-based attenuation correction (MR-AC). Most groups have proposed MR-AC algorithms for brain PET studies and more recently also for torso PET/MR imaging. Most MR-AC strategies require the use of complementary MR and transmission images, or morphology templates generated from transmission images. We review and discuss these algorithms and point out challenges for using MR-AC in clinical routine.

Discussion MR-AC is work-in-progress with potentially promising results from a template-based approach applicable to both brain and torso imaging. While efforts are ongoing in making clinically viable MR-AC fully automatic, further studies are required to realize the potential benefits of MRbased motion compensation and partial volume correction of the PET data.

Keywords PET/MRI PET quantification .

Attenuation correction

\section{Introduction}

Combined PET/CT has emerged as a powerful imaging modality for the diagnosis, staging and restaging of a variety of cancers [1]. Less frequently perhaps, PET/CT is being used for cardiology and neurology examinations. In general, $\mathrm{PET} / \mathrm{CT}$ examinations provide complementary and intrinsically coregistered CT and PET image volumes, whereby the CT transmission data are also used routinely for attenuation correction (AC) [2]. In general, CT-based AC (CT-AC) is based on a piecewise linear scaling algorithm that translates CT attenuation values into linear attenuation coefficients at 
$511 \mathrm{keV}[3,4]$. By using the CT images for the purpose of AC, lengthy PET transmission scanning with conventional rod- or point sources (TX-AC) has become obsolete in commercially available PET/CT tomographs.

Recently, a combination of PET and MRI has been proposed as a promising alternative to existing dual modality PET/CT systems, and the first images of patients were presented in late 2006 [5]. The realization of PET/MR tomographs beyond small-animal imaging prototypes $[6,7]$, however, remains challenging. In particular, the lack of conventional or X-ray transmission sources mandates alternative approaches to AC of the complementary emission data [8].

Since current concepts of combined PET/MR tomographs do not allow separate CT-like transmission sources, PET attenuation coefficients need to be calculated from the available MR images. CT images are required at effective CT energies of $70-80 \mathrm{keV}$ and represent the pixel-wise distribution of attenuation coefficients and, thus, yield a measure of the electron density in the image volume. In contrast, MR images reflect the distribution of hydrogen nuclei (Fig. 1). Thus, MR-based AC (MR-AC) is far more challenging than CT-AC since MR image voxel values correlate with the density of hydrogen nuclei in tissues and tissue relaxation properties rather than with the electron density-related mass attenuation coefficients of these tissues. Therefore, a direct mapping of CT-like attenuation values from available MR images is challenging [9].

Although preclinical PET/MR prototype systems [10] have been around since the early 1990's, MR-AC is still work in progress. While early preclinical PET/MR design concepts did not include means for $\mathrm{AC}[6,7]$, a relatively simple two-class AC scheme was suggested for the first clinical prototype [5]. The lack of viable MR-AC methods today can be explained by the fact that attenuation is less critical in small animals than in patients, and, therefore, the issue of AC was of less importance in preclinical PET and PET/MRI.

As the recognition of combined PET/MR imaging is increasing we review the status of estimating PET attenuation maps from available MR images in clinical PET/MR imaging scenarios. We discuss potential pitfalls of MR-AC as well as a number of advantages inherent to MR-AC that could make it more useful than CT-AC where applicable.

\section{Methods}

Table 1 summarizes the main approaches to MR-AC for imaging patients. Interestingly, several studies on MR$\mathrm{AC}$ appeared when such combined devices were not yet considered seriously for clinical use. These early studies were focused on applications in the brain PET [11, 12]. With the considerations of clinical PET/MR prototypes several groups have proposed algorithms for extracranial MR-AC as well $[9,13]$.

\section{Brain imaging}

Segmentation approaches MR-AC for brain applications was first addressed by Le Goff-Rougetet et al., who proposed a method to calculate PET AC factors from MR images in clinical examinations when both PET and MRI were required [11]. They argued that MR-AC helps simplify the clinical protocol and reduces the patient dose from standard PET transmission scanning. Their methodology, which they first applied to an FDG/water-filled, cylindrical Lucite phantom, is based on a coregistration of the MR images to the PET transmission images using a surface matching technique. The coregistered MR images are then segmented into three classes (Table 1). Air is considered only outside the patient. Appropriate linear attenuation coefficient values $(\mu)$ at $511 \mathrm{keV}$ are then assigned to these tissue classes.

El Fakhri et al. [14] also mentioned MR-AC, but they did not provide further details of their implementation or a performance evaluation. In a personal communication the authors stated that they acquired two MR sequences for each subject and performed a cluster identification on the joint histogram prior to assigning the corresponding attenuation values.
Fig. 1 Axial MR (a) and CT (b) images of the neck of the same patient showing the differences in appearance of bone, air (trachea) and soft tissue. The inability to separate bone and air-filled structures clearly on the MR image renders piecewise linear scaling approaches to AC impossible
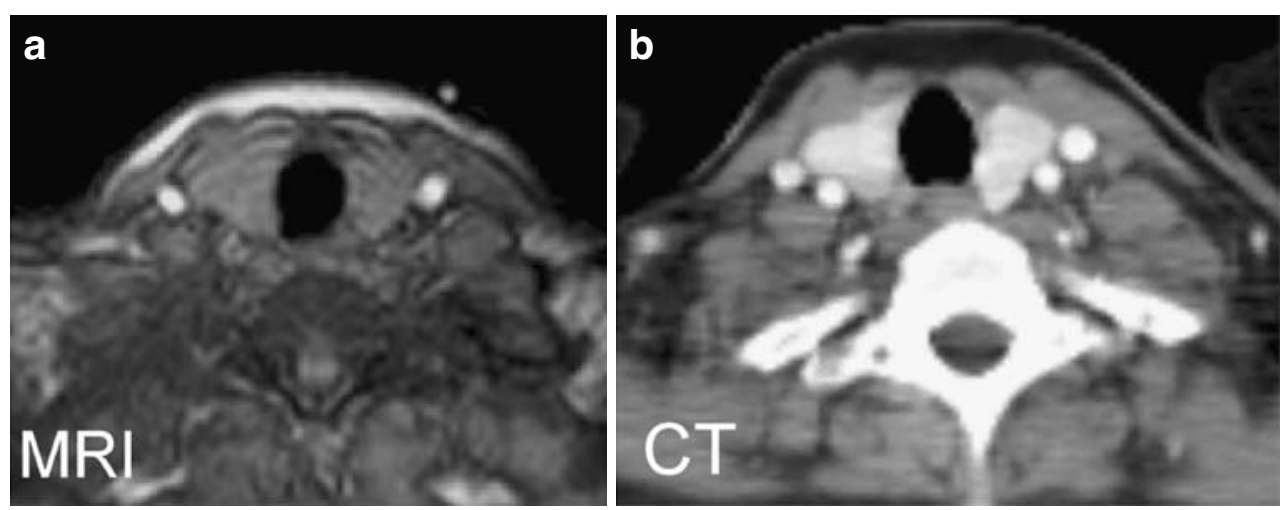
Table 1 Overview of studies on MR-AC

\begin{tabular}{|c|c|c|c|}
\hline Area & Methodology & Results & Reference \\
\hline Head & $\begin{array}{l}\text { Phantom studies and one patient; } 0.5-\mathrm{T} \text { MRI with } \mathrm{T} 1-\mathrm{W} \text {; } \\
\text { surface-based, linear coregistration of } \mathrm{PET}_{\mathrm{TXAC}} \text { and MRI, } \\
\text { three-class MR segmentation and mu-assignment (skin } \\
0.095 \mathrm{~cm}^{-1} \text {, brain } 0.095 \mathrm{~cm}^{-1} \text {, bone } 0.151 \mathrm{~cm}^{-1} \text { ) }\end{array}$ & $\begin{array}{l}\text { Maximum difference between } \mathrm{PET}_{\mathrm{TXAC}} \\
\text { and } \mathrm{PET}_{\mathrm{MRAC}} \text { was } 11 \% \text { and } 12 \% \text { in } \\
\text { phantoms and patient, respectively. Future: } \\
\text { investigate coregistration of emission-only } \\
\text { and MRI; to improve MR segmentation of } \\
\text { the lower skull }\end{array}$ & 11 \\
\hline Head & $\begin{array}{l}\text { Ten patients; } 1.5 \text {-T MRI with T1-W; linear coregistration of } \\
\text { PET }_{\text {FEAC (fitted ellipse AC) and MRI, five-class fuzzy- }} \\
\text { information segmentation of MRI and mu-assignment } \\
\text { (air } 0 \mathrm{~cm}^{-1} \text {, brain } 0.0993 \mathrm{~cm}^{-1} \text {, skull } 0.143 \mathrm{~cm}^{-1} \text {, nasal } \\
\text { sinuses } 0.0553 \mathrm{~cm}^{-1} \text {, scalp not considered) }\end{array}$ & $\begin{array}{l}R^{2}=0.91 \text { for ROIs in } \text { PET }_{\mathrm{TXAC}} \text { and } \mathrm{PET}_{\mathrm{MRAC}} \text {. } \\
\text { Improvement in PET image quality following } \\
\text { MR-AC. Future: integrate MR information into a } \\
\text { unified statistical PET reconstruction method }\end{array}$ & 12 \\
\hline Head & $\begin{array}{l}\text { Four subjects; } 1.5-\mathrm{T} \text { MRI with T1-W; linear coregistration } \\
\text { of MRI to TX and four-class segmentation/mu-assignment } \\
\text { (brain } 0.099 \mathrm{~cm}^{-1} \text {, bone } 0.146 \mathrm{~cm}^{-1} \text {, sinus area } 0.054 \mathrm{~cm}^{-1} \text {, } \\
\text { soft tissue } 0.095 \mathrm{~cm}^{-1} \text { ). Suggest also a } \\
\text { template-based AC (T-AC) with MR and TX data } \\
\text { warped to SPM2 templates and using measured } \\
\text { TX-based mu for AC. }\end{array}$ & $\begin{array}{l}\text { Maximum difference between } \mathrm{PET}_{\mathrm{MRAC}} \text { and } \\
\mathrm{PET}_{\mathrm{TXAC}} \text { was } 9 \% \text { in occipital cortex. Somewhat } \\
\text { smaller differences from T-AC. Future: investigate } \\
\text { gender-specific templates for T-AC }\end{array}$ & 17 \\
\hline Torso & $\begin{array}{l}\text { Ten whole-body patients; } 1.5-\mathrm{T} \text { MRI with T1-W; nonlinear } \\
\text { coregistration of CT and MRI and CT-MR histogram } \\
\text { matching }\end{array}$ & $\begin{array}{l}\text { MR-AC challenged by differences in patient } \\
\text { positioning, lack of MR surface coils and } \\
\text { coregistration accuracy. Future: complementary } \\
\text { PET/CT and MRI datasets allow evaluation of } \\
\text { singular steps in MR-AC when comparing to } \\
\text { CT-AC as gold standard. }\end{array}$ & 9 \\
\hline Head, torso & $\begin{array}{l}\text { Head: three patients scanned on PET/CT and } 1.5-\mathrm{T} \text { MRI } \\
\text { with T1-W; linear coregistration of MR-CT. Pseudo-CT } \\
\text { prediction via atlas/machine learning method using } 17 \\
\text { coregistered MR-CT pairs as training data. Torso: five } \\
\text { whole-body patients; } 1.5-\mathrm{T} \text { MRI with T1-W, nonlinear } \\
\text { coregistration (ongoing study) }\end{array}$ & $\begin{array}{l}\text { Head: mean absolute difference } \text { PET }_{\text {MRAC }} \text { to } \\
\text { PET }_{\text {CTAC was } 3.2 \% \text {, maximum error } 10 \% \text {. Torso: }} \\
\text { initial results show principal feasibility of predicting } \\
\text { bone attenuation from MRI. Future: head, more } \\
\text { suitable higher resolution MRI; torso, large } \\
\text { whole-body database with dual contrast MR }\end{array}$ & 13,18 \\
\hline
\end{tabular}

All studies compared PET with standard PET AC (TX-AC) and MR-AC. Note: different studies used different reconstruction and correction techniques and, therefore, the results may not be directly comparable.

$m u$ linear attenuation coefficient at $511 \mathrm{keV}, T 1-W$ T1-weighted MRI sequence, $T X$ transmission scan, $T-A C$ template-based AC, Future extension of the study-related research as proposed by the authors.

An alternative method for MR-AC in brain PET was suggested by Zaidi et al. [12]. The authors had previously shown that the quality of PET neurology imaging was insufficient when standard PET AC methods were applied [15]. Therefore, they studied the use of MR-AC in brain PET (Table 1). They present a workflow based on the availability of coregistered PET images, following standard (ellipse-fitted) AC, and MR images [12]. Using a segmentation method based on fuzzy logic the coregistered MR images are segmented into five tissue classes that are assigned attenuation coefficients at $511 \mathrm{keV}$. The entire process for MR-AC was reported to take $10 \mathrm{~min}$ on a Sun SPARC with minimal user intervention. Like Le Goff-Rougetet et al., the authors account for the head holder before using the segmented MR-based attenuation map for MR-AC.
Atlas approaches A viable alternative to multistep segmentation procedures $[12,16]$ is to use atlas co-registration (Fig. 2). For MR-AC, an atlas typically consists of a template MR image together with a corresponding attenuation label image. The template MR image can be obtained as an average of co-registered MR images from several patients. The label image could represent a segmentation into different tissue classes (e.g. air, bone and soft tissue) or a coregistered attenuation map from a PET transmission scan or a CT scan with continuous attenuation values. The template MR is warped to the patient-specific MR image volume. When applying the same spatial transformation to the atlas attenuation image a corresponding patient-specific attenuation map is generated. 
Fig. 2 Principle of atlas-based MR-AC. The atlas consists of a matching MR-CT image volume that can be generated from a patient. An atlas of attenuation values at $511 \mathrm{keV}$ is generated from matching $\mathrm{CT}$ images. The atlas MR image (top left) is coregistered to the MR image volume of a specific patient (bottom left). This transformation is applied to the corresponding CT atlas, thus generating an attenuation image (i.e. pseudo-CT image) that approximately matches the patient anatomy
Matched MR-CT Pair = Atlas

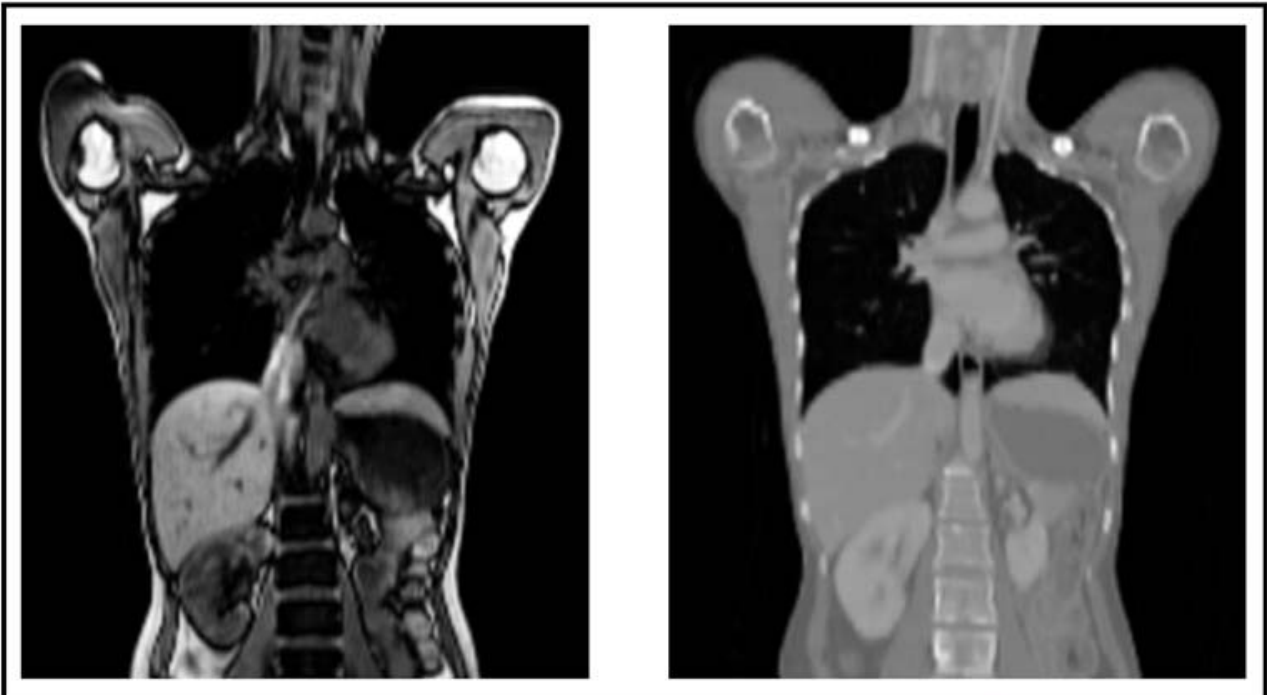

Determine registration Atlas-MR to Patient-MR

Apply registration to Atlas-CT

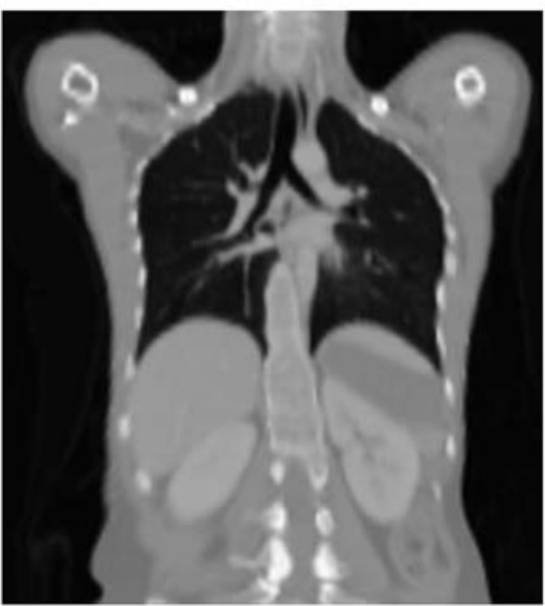

MR-derived Pseudo-CT
Atlas-based approaches to MR-AC were presented by Kops and Herzog [17] and Hofmann et al. [18]. Kops and Herzog generate a template of PET transmission images from the data from 10 patients that is matched to the PET transmission template within SPM2 [19]. The MR template within SPM2 (which is already aligned with the PET transmission template) is normalized to the MR image of the patient. The obtained transformation is then applied to the template attenuation image to yield an attenuation image for this patient. The same group has also employed MR-AC based on an MR segmentation method by Dogdas et al. [16] following linear coregistration of the MR image to the measured PET transmission image.
Hofmann et al. have suggested a revised atlas approach to MR-AC [18]. Here, the authors utilize a set of aligned MR-CT image volumes of 17 patients. Each of the available $17 \mathrm{MR}$ image volumes from the MR-CT pairs is coregistered to the MR image volume from the PET/MR study. The coregistration vectors are applied to the corresponding CT image volumes thus generating $17 \mathrm{CT}$ image sets that are aligned to the MR image set from the patient. Subsequently, a pattern recognition approach is used to match the MR image of the patient with the appropriate CT information from the MR-CT dataset that best matches the patient information. This voxel-based approach can merge partial subvolumes from independent 
datasets into a single CT volume that is used for MR-AC of the patients. This atlas-based algorithm was validated on three clinical datasets comparing MR-AC to the gold standard CT-AC [18].

\section{Torso imaging}

Due to the lack of prototype systems for whole-body PET/ MRI studies of MR-AC, algorithms for extracranial applications are scarce. Beyer et al. set up a toolbox that facilitates cross-validation of MR-AC and CT-AC using matching PET/CT and MR image volumes [9] from 10 patients. They studied ten patients who underwent routine torso scans with arms up on a combined PET/CT tomograph. Within 1 day of the PET/CT examination, complementary MR scans were acquired. MR imaging was performed on a 1.5-T system with patients positioned with their arms down. Single-station, transverse T1-weighted VIBE MR images were used to generate pseudo-CT images. First, the MR images were coregistered to the CT images using nonlinear curvature-regularized coregistration in conjunction with mutual information. Second, the MR voxel value intensity distribution was matched to that of the coregistered CT image. MR-CT intensity transformation was performed in a three-step process based on a nonproprietary histogram-matching algorithm. PET images were reconstructed on the PET/CT console following AC based on CT transmission images ( PET $_{\mathrm{CTAC}}$ ) and MRbased pseudo-CT images (PET MRAC) $_{\text {. }}$.

Although predominantly used for brain imaging, atlasbased methods can also be applied to whole-body imaging. However, anatomic variability is high and it is unlikely that a general spatial transformation captures all variables between a template and patient-specific anatomy. Hofmann et al. [18] presented a machine learning approach that combines the information from an atlas registration with local information that is extracted from small image patches. Thus this method is less dependent on accurate template-to-patient registration. Validation was performed on two whole-body rabbit datasets and, in ongoing work, on five human torso datasets [13].

\section{Results}

MR-AC can be evaluated by comparing the PET images obtained following standard TX-AC, CT-AC and MR-AC. We refer below to these images as PET $_{\text {TXAC }}$, PET $_{\text {CTAC }}$ and PET $_{\text {MRAC }}$, respectively.

Both TX-AC and CT-AC have their shortcomings. Depending on the scan time, TX scans have relatively high noise levels that can be detrimental to AC. CT scans on the other hand have very low noise levels, but the mapping from CT Hounsfield units to $511 \mathrm{keV}$ attenuation values can be incorrect, particularly in the case of inorganic materials such as metal implants. Despite these problems, both TX-AC and CT-AC are commonly used and accepted. In accordance with the literature we present both PET $_{\text {TXAC }}$ and $\mathrm{PET}_{\mathrm{CTAC}}$ as the gold standard against which $\mathrm{PET}_{\mathrm{MRAC}}$ should be compared.

The comparison can be done visually or quantitatively by means of relative differences of the reconstructed PET activity distributions. Differences can be assessed on a voxel-by-voxel basis, or perhaps more commonly for regions of interest (ROI). ROIs can be defined automatically or manually by a human expert. For a study with $n$ patients, where $p$ ROIs are defined for each patient, it is impractical to quote all $n$ times $p$ differences. Therefore, it is preferred to report either the maximum differences or the mean absolute difference across all voxels. Some authors have quoted the mean differences, where the mean was taken from the positive or negative differences. This value only indicates the existence of an overall bias in the method; a value of zero for the differences would merely indicate that activity was overestimated as often as it was underestimated.

For a summary of the results of the most significant studies on MR-AC see Table 1.

\section{Brain imaging}

Le Goff-Rougetet et al. [11] evaluated their MR-AC method for phantoms and a patient scan. Using ROIs within selected axial images of the phantom they found a maximum difference of $11 \%$ between $\mathrm{PET}_{\mathrm{MRAC}}$ and $\mathrm{PET}_{\mathrm{TXAC}}$. The patient study revealed a maximum difference of $12 \%$, primarily in the occipital cortex. In the study by Le Goff-Rougetet et al. an expert placed ROIs into different regions of the brain of the coregistered MR images. However, ROIs were not placed in the lowest and highest slice of the MRI volume as their MR segmentation method still needed refinement for these areas.

Figure 3 shows brain images of an FDG-PET study by Zaidi et al. [12] comparing segmented MR-AC with a standard, transmission-guided AC. The quality of the PET images following MR-AC appeared somewhat improved, which could be attributed to the lower noise levels in the MR-based attenuation maps (Fig. 3b). Analysis of the differences between the two methods of $\mathrm{AC}$ was performed across 10 patient sets. Despite a tendency of the method to lead to activity overestimation, overall correlation of ROI activity values on PET $_{\text {MRAC }}$ and PET $_{\text {TXAC }}$ was good $\left(r^{2}=\right.$ 0.91 ), indicating the feasibility of segmented MR-AC as suggested by the authors (Table 1).

Kops and Herzog [17] validated their MR-AC algorithm in four patients (Fig. 4). An analysis of ROIs drawn 


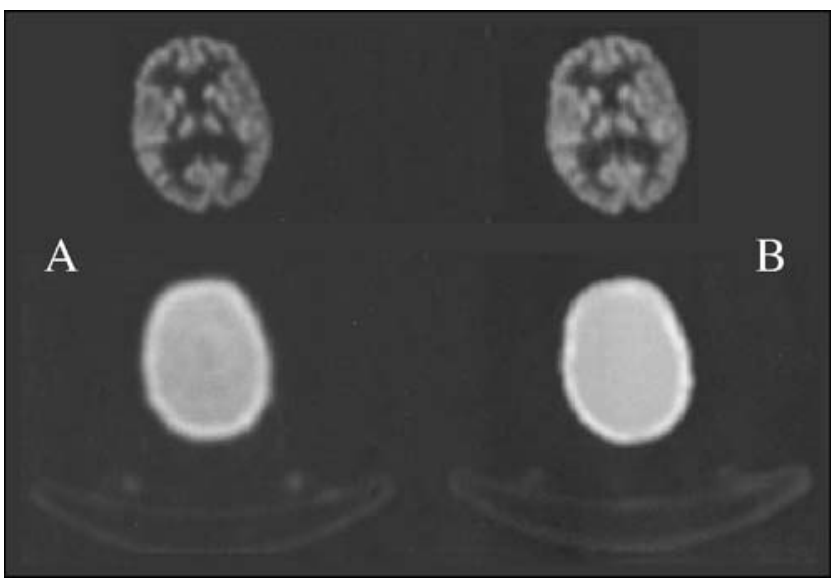

Fig. 3 MR-AC for brain PET [12]. Axial slices through an FDG-PET scan of a brain of a patient following standard AC (a) and MR-AC (b) with the PET images (top) and the corresponding attenuation maps (bottom). Note the patient bed and head holder prior to MR-AC. The PET image following MR-AC appears visually similar with a slightly better signal-to-noise ratio

on cortical and subcortical structures demonstrated that PET $_{\text {MRAC }}$ differed from PET $_{\text {TXAC }}$ by less than $10 \%$. Maximum differences were observed again in the occipital cortex and in the caudate nucleus. The same group also
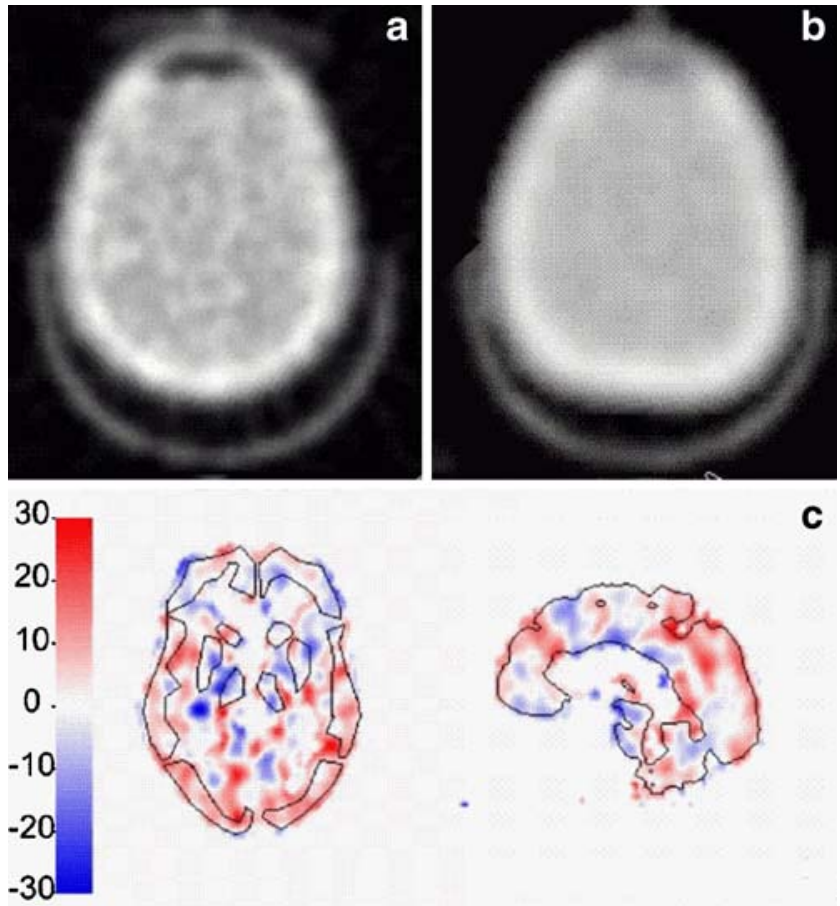

C

Fig. 4 MR-AC for brain PET: template-based AC. a Attenuation map measured through a PET transmission scan. b Attenuation map obtained through template coregistration and addition of the head holder. c Voxel-by-voxel calculation of relative differences between PET attenuation corrected using attenuation maps $\mathbf{a}$ and $\mathbf{b}$. (images from Kops and Herzog [12]) evaluated a template-based $\mathrm{AC}$, which resulted in a $9 \%$ difference from PET $_{\text {TXAC. }}$.

Hofmann et al. [18] evaluated their method with three patient scans. Automated ROI analysis of $\mathrm{PET}_{\mathrm{MRAC}}$ and PET $_{\text {CTAC }}$ yielded a mean absolute difference of $3 \%$ and a maximum difference of $10 \%$, which is similar to the previous MR-AC approaches to brain PET (Fig. 5).

\section{Torso imaging}

Data on the utility of MR-AC for torso or whole-body applications are still sparse. Beyer et al. [9] presented a setup that allows comparison of $\mathrm{PET}_{\mathrm{MRAC}}$ and $\mathrm{PET}_{\mathrm{CTAC}}$ using datasets from the same patients (Fig. 6). However, in reality such complementary datasets are not available from PET/ MR studies and, therefore, this toolbox can be used only as guidance in the evaluation of pitfalls in MR-AC. Nonetheless, the authors were able to demonstrate that histogram matching is a feasible technique to transform MR to pseudo-CT attenuation values if the MR image quality is high and MR images are free of distortions. In those cases where the MR image is distorted the PET images will be affected by MR-AC. The study illustrates the need for accurate patient positioning between MRI and PET scans without quantifying these effects further.

In an ongoing study, Hofmann et al. [13] aim to use an atlas-based approach to MR-AC that is applicable to the human torso, and possibly whole-body imaging. Their method can predict bone structures on MR sequences that typically do not allow intensity-based segmentation of bone. Figure 7 shows the initial results from atlas-based MR-AC for areas outside the brain.

\section{Discussion}

Various approaches for predicting the attenuation maps from MR images on PET/MRI examinations of patients are reviewed. While segmentation-based approaches work well for brain applications, torso imaging with PET/MRI may require more sophisticated methodologies such as atlas-based image transformations from MR images to pseudo-CT images. In general, MR-AC not only needs to address adequate transformation of MRI pixel value information to appropriate PET attenuation values, but also, in order to be viable in clinical routine. needs to account for additional pitfalls in torso and whole-body imaging. The pitfalls include the accurate representation of bone (typically not seen on MR images), potential truncation effects from patients extending beyond the transverse field-of-view (FOV) of the MR system and the presence of MR surface coils typically not seen on MR imaging. 
Fig. 5 MR-AC for brain PET using the template-based method of Hofmann et al. [18]. Axial slices through patient data with mid-plane sections (top) and lower brain sections (bottom). T1-W spin-echo MR images (a), pseudo-CT images (b) as predicted from the template-based MR-AC, and original CT images (c). Note the visual similarity between the pseudo$\mathrm{CT}$ and the original CT images. The T1-W spin-echo MR images used here were acquired with a comparatively large slice spacing of $4 \mathrm{~mm}$, which may explain the limited improvement in overall image quality, although the accuracy improvement within a given axial slice was high
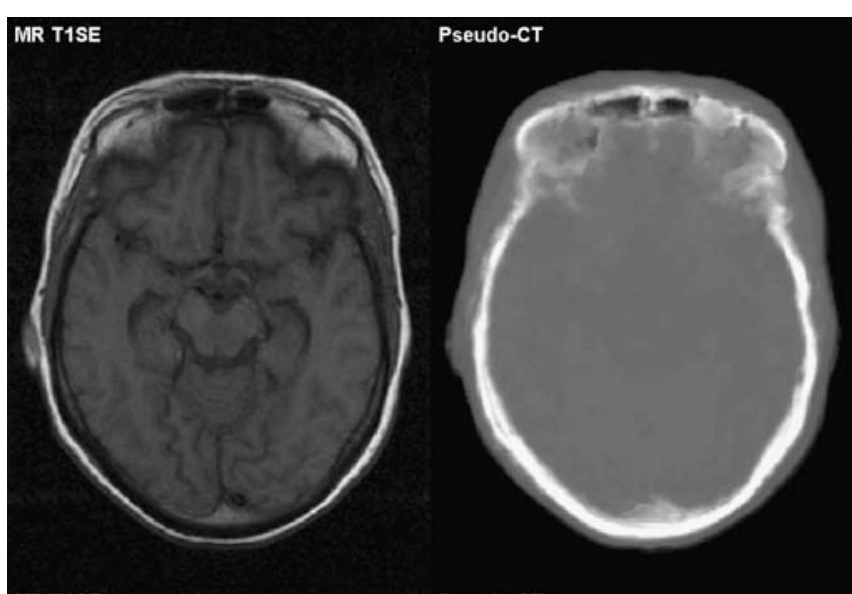

Real CT

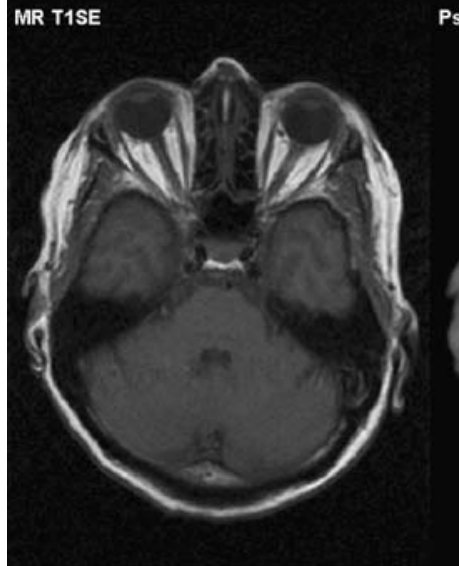

A

Pseudo-CT

Real CT

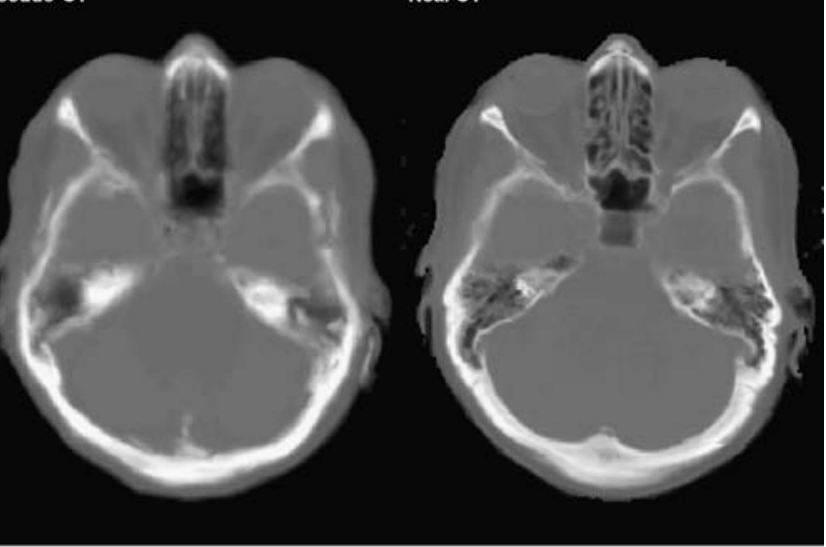

B

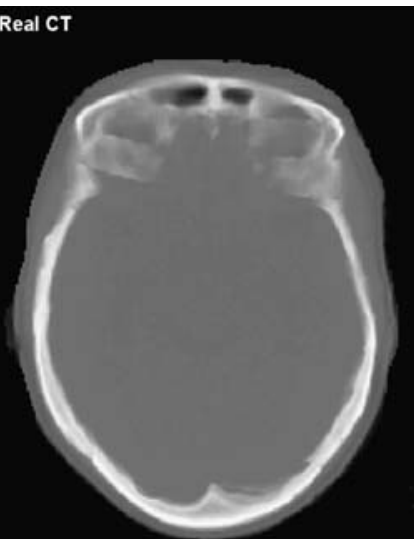

The presence of bone

As bone structures are difficult to separate on MR images a straightforward approach to MR-AC would be to simply ignore bone. This is not a new approach, and was shown in early studies on CT-AC to be of less impact than originally expected $[20,21]$ despite the fact that the fraction of cortical bone varies in axial images.

Figure 7 shows an example of CT-AC performed with and without consideration of bone. This example illustrates the minimum bias expected from ignoring bone attenuation and considering this tissue class as soft tissue. In practice, an MR$\mathrm{AC}$ algorithm that ignores bone tissue may also falsely attribute air as soft tissue (Fig. 1) and thus introduce a much higher bias. In a conference abstract Martínez-Möller et al. [22] recently reported a patient PET study processed with MR-AC without consideration of bone compared with CTAC. Based on an ROI analysis the authors reported mean differences of $1.7 \%, 7.3 \%$ and $2.9 \%$ for lung, bone and neck lesions, respectively. They concluded that MR-AC without accounting for bone tissue does not lead to a clinical bias. However, further studies are required to estimate the bias on uptake in bone metastasis in case MR-AC is performed without considering the presence of cortical bone.
MR imaging with ultrashort echo time (UTE)

Instead of performing advanced segmentation methods on standard MR images one may utilize dedicated MR sequences, such as ultrashort echo time (UTE) sequences [23, 24] that yield signal even from cortical bone (Fig. 8). Typically, the use of just a single UTE image does not enable bone to be separated from non-bone tissues. However, when combined with a late echo image it is, in principle, possible to detect bone as that structure that yields signal on the short echo image, but not on the late echo image (Fig. 8). By using multiecho sequences [25] the two images can be acquired in one scan. While it seems promising for brain applications, it may not be acceptable as part of whole-body imaging protocols since the acquisition time is on the order of several minutes per bed position.

\section{Required PET accuracy}

Which of the presented MR-AC methods will be accepted in clinical routine will depend not only on which MR-AC method yields the highest accuracy, but also the accuracy considered sufficient for clinical work. If, for example, variations of up to $10 \%$ in PET activity values are considered 
Fig. 6 MR-AC for torso applications [9]. From top to bottom: CT images from PET/CT studies are coregistered to the available MR images. CT-MR histogram matching yields images with pseudo-CT attenuation values that are used for MR-AC. PET images following CT-AC and MR-AC show major differences if the MR images inherit artefacts from suboptimal imaging protocols. If the MR image quality is good (thorax) and the coregistration is accurate, MR$\mathrm{AC}$ based on histogram matching appears feasible

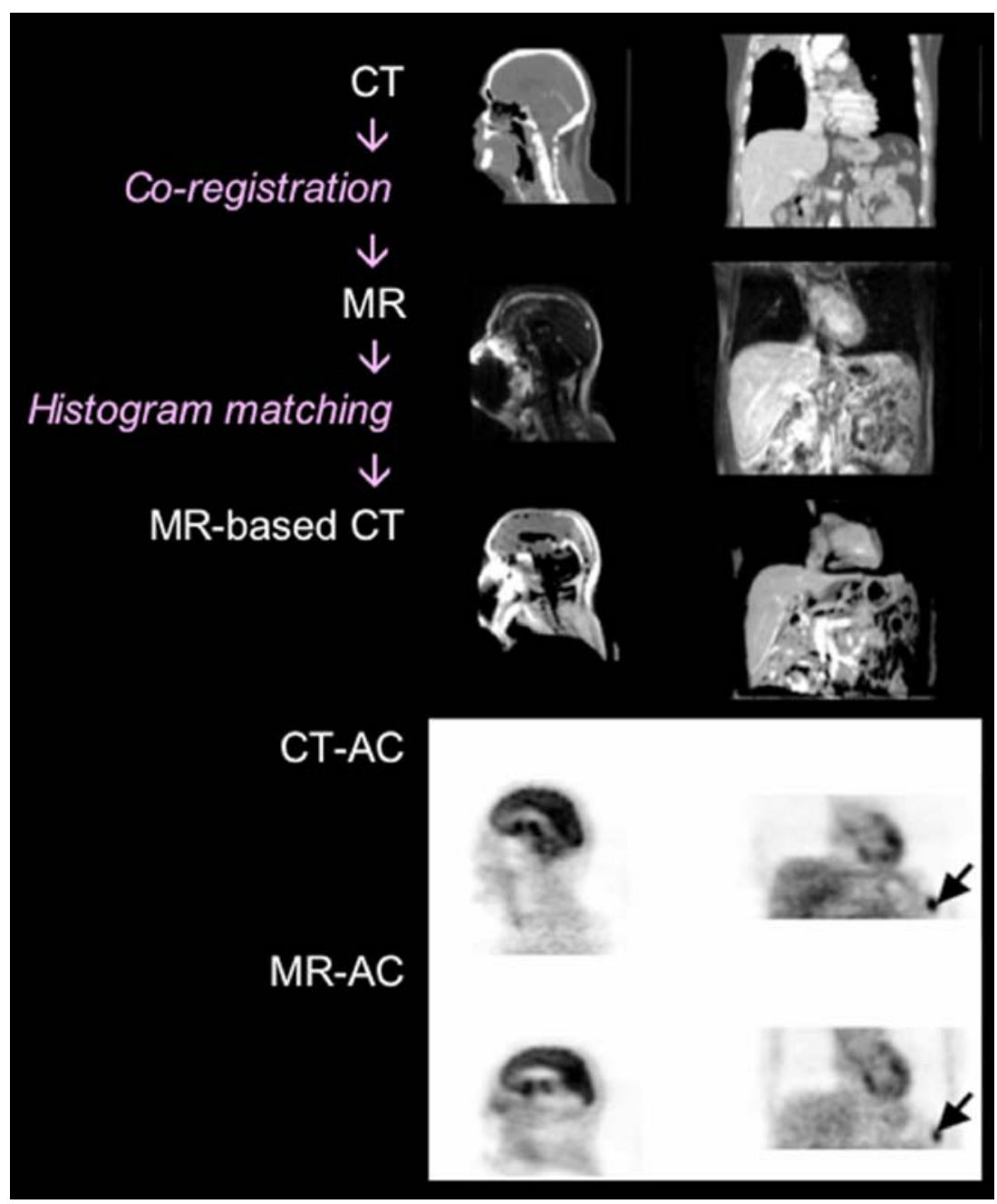

acceptable in clinical routine, then methods with higher accuracy might be dismissed while giving preference to methods with other advantages such as robustness or computational speed. PET uptake values, such as the measured standard uptake values (SUV), are affected by many factors such as uptake time, body composition, glucose load and others that are independent of the imaging device [26].

\section{Validation of MR-AC methods}

In the absence of simultaneous PET/MR systems, validation of whole-body MR-AC is inherently difficult; movement of the patient between $\mathrm{MR}$ and PET(/CT) examinations is unavoidable. Therefore, even if the attenuation map could be predicted with a high accuracy from the MR image, patient movement between the MR scan and a TX or CT scan may still cause the MR-predicted attenuation map to be different from the reference scan attenuation values, thereby also causing a difference

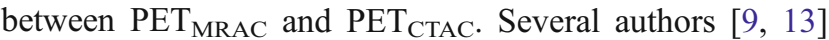
have suggested compensating for patient movement be- tween scans by performing nonrigid MR-CT coregistration. It should, however, be noted that not all motion-related misalignments can be corrected. For example, pockets of gas in the abdominal region may vary significantly between scans, or not even appear on one of the two complementary studies. In addition, the validation of nonlinear coregistration algorithms remains an open issue [27] that requires addressing if coregistration is to become an integral part of routine MR-AC.

\section{Truncated field-of-view}

In clinical PET/MR imaging patients may well extend beyond the transverse FOV of the MRI system. Thus, the arms and even the trunk of the patient may not be fully covered by the MR image. Nonetheless, the contribution of the truncated anatomy to overall attenuation needs to be accounted for. The very same problem was described for PET/CT applications where truncated attenuation maps were shown to yield significant image distortion and bias near the area of truncation [28-31]. Recently, Delso et al. 


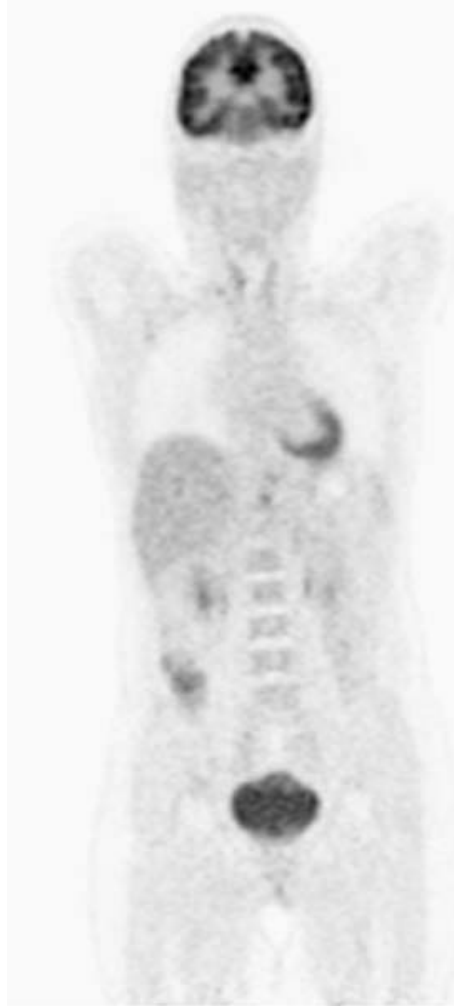

A

Fig. 7 Effect of ignoring cortical bone during CT-AC. a PET image reconstructed using the original CT image. b PET image reconstructed using the same CT image with all bone structures set to the attenuation value of soft tissue, thus simulating a best-case scenario of MR-AC

discussed the effect of MR truncation on MR-AC [32]. Their study showed that when the arms were outside the FOV the PET activity after AC was biased by up to $14 \%$ in that area [32]. Using simple image processing techniques they were able to recover the arms in the truncated image and thus reduce the quantification bias to $2 \%$.

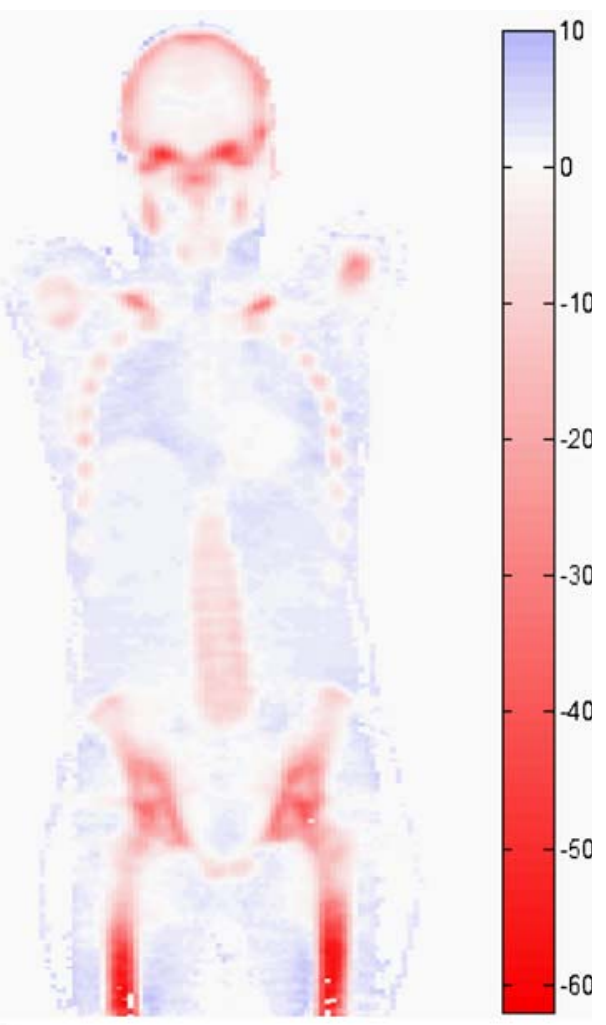

C

where bone attenuation is ignored. $\mathbf{c}$ Relative difference (\%) between $\mathbf{a}$ and $\mathbf{b}$ showing that the largest effect is in the skeleton. Note voxels are set to white in low uptake regions with SUV $<0.2$ in the original PET image

An alternative solution would be to use the uncorrected PET image to estimate the patient cross-section in those areas outside the measured FOV where no MR information is available. The feasibility of such an approach still needs to be validated. In imaging scenarios with highly specific tracers the arms may be difficult to segment automatically

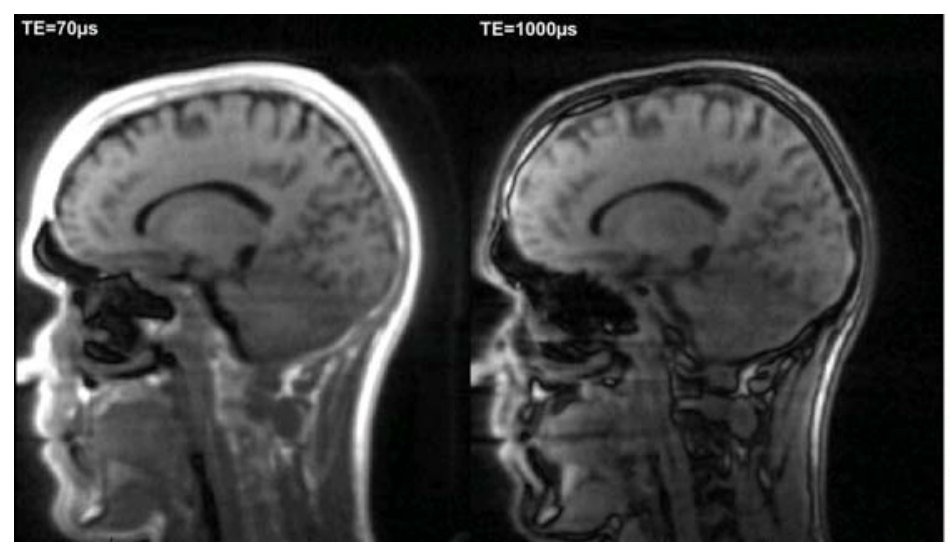

A

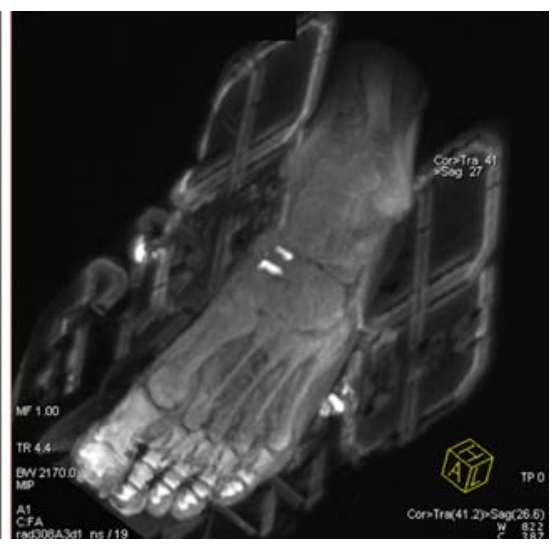

B

Fig. 8 MR images acquired with a 3-D UTE sequence. a Sagittal brain section acquired with short echo (0.07 ms left $)$ and late echo (1 ms right). b Angled view of a human foot. Note that bone yields a high signal as do parts of the coil housing 
in the uncorrected PET images. Yet another approach would be to predict the body cross-section through atlas matching outside the FOV of the MR image. In theory, these approaches could even be combined such that the atlas co-registration is performed based on the MR image where the MR image is available, and elsewhere based on the uncorrected PET image.

\section{MR coils}

The fact that the MR coils are located inside the FOV of the PET system is a challenge that has not yet been addressed by any of the studies on MR-AC. For brain scans, the head coil is rigid and its attenuation values can be estimated from a baseline CT scan. Subsequently for any PET/MR study only the relative position of the head coil inside the PET/MR system would be required. For extracranial examinations the situation is far more difficult. Surface coils are required to avoid suboptimal signal generation (Fig. 6). Surface coils contain elastic components and hence cannot be located easily with respect to the gradient coil or the patient. MR sequences with UTE could possibly help detect surface coil landmarks and thus help account for their attenuation.

\section{User intervention}

Ideally, for application in clinical PET/MRI scenarios MRAC should be fully automatic in order to limit user interaction and, subsequently, examination and processing times. Despite claims of some groups that their method for MR-AC is "robust", problems remain that require "some manual intervention of the operator" [12]. Thus, automation of MR-AC remains a challenge, particularly in patients with a large deviation from normal anatomy.

Potential benefits of MR-AC

In PET/CT the PET image is acquired over several minutes, while the CT scan is a matter of seconds and is frequently acquired during a single breath-hold. As a result, patient motion typically causes local misalignment between the PET and $\mathrm{CT}$ images and may lead to serious artefacts for $\mathrm{AC}$, for example near the diaphragm [31]. Some authors have recommended 4-D PET/CT acquisition and $\mathrm{AC}[33,34]$; however, this may involve a substantially higher patient radiation dose.

As an MRI examination typically takes much longer than a CT exam, patients conceivably spend an even longer time in an PET/MRI system than in a PET/CT. Consequently, patient motion is likely to cause even more severe artefacts in PET/MR than in PET/CT. Here, the use of periodic MRI navigator signals in conjunction with a 4-D model of the human torso may help to correct for motioninduced image degradation in PET/MR data following 4-D MR-AC, which would be a major advantage over CT-AC.

\section{Additional potential benefits of simultaneous PET/MR acquisition}

As early as 1991, Leahy et al. [35] suggested that PET reconstruction could be improved by using anatomical MR images from the same patient as prior information. This remains a field of active research and the potential of the
Fig. 9 Comparison of a standard filtered back projection $(F B P)$ that was reconstructed PET image, and a PET image that was constructed using a maximum a posteriori $(M A P)$ approach with an MR image from the same patient used as prior information (images with permission from Nuyts et al. [37])
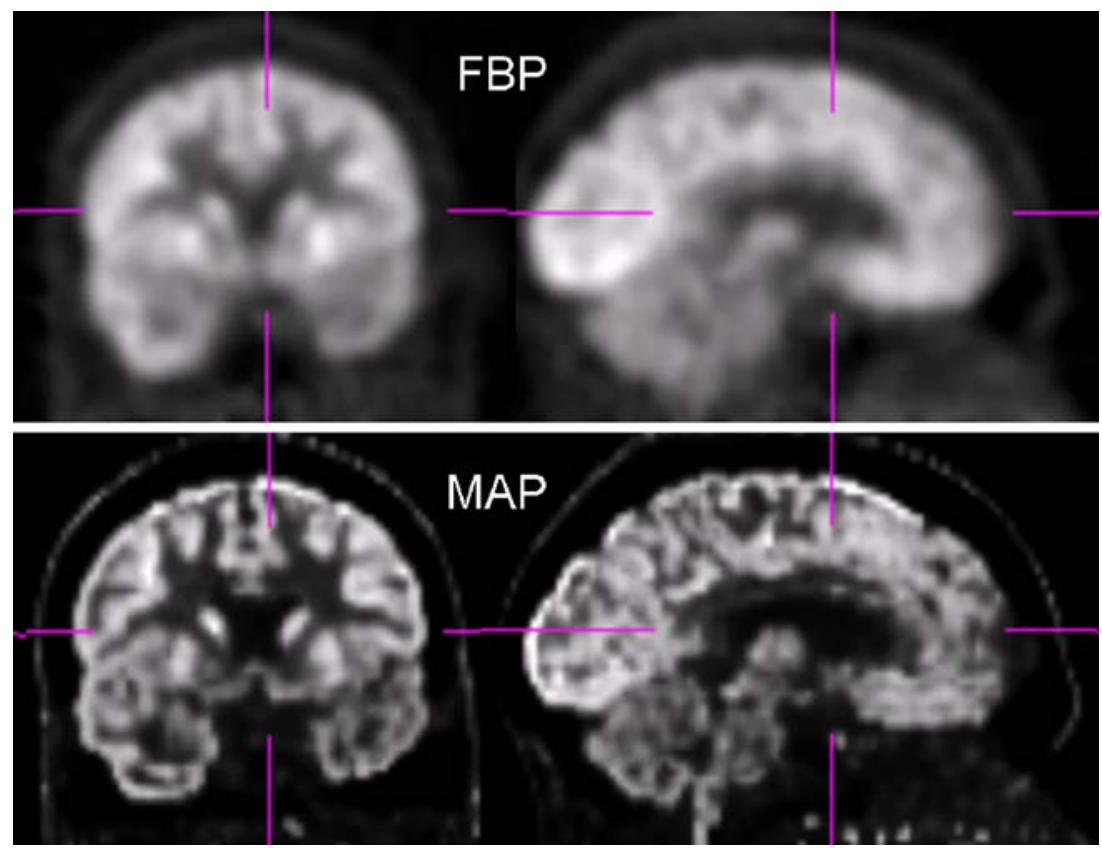
method can be seen in Fig. 9. While it is commonplace today that almost all neurology patients who receive a PET scan also receive an MR scan, MR-guided PET reconstruction has not yet made the transition from research into clinical routine. Aside from logistical problems of automatically retrieving the matching MR image from the PACS, one of the reasons for this might be that misregistrations, which are unavoidable in retrospective PET-MR coregistration, quickly lead to deterioration of image quality [36]. In combined PET/MR tomographs, the coregistration accuracy is improved and may help promote the concept of MR-guided PET image reconstruction.

Even if the PET image is reconstructed independently of the MR image, it is still possible to use the MR image of the patient as an aid for improved quantification. In particular MR-guided partial volume correction (PVC) was suggested as early as in 1990 [38, 39]. Again PET and MR images from combined PET/MRI examinations may facilitate improvements in MR-based PET quantification through the use of MR-based PVC.

\section{Conclusion}

With the onset of a research interest in combined PET/MR imaging several studies have appeared on the use of MR for AC of the PET data. MR-AC is not as straightforward as CT$\mathrm{AC}$ that allows the estimation of $511 \mathrm{keV}$-attenuation maps from CT transmission images. In the absence of CT-like transmission sources in PET/MR alternative solutions to MR$\mathrm{AC}$ include the use of complex segmentation tools that were shown to work for brain applications. In extra-cranial PET/ MR other approaches that include atlas-matching appear more promising. While MR-AC is work-in-progress further advantages of MR-AC over CT-AC become apparent, which include the additional use of MR for retrospective motion correction or partial volume correction of the PET.

Conflict of interest statement Bernd Pichler is a consultant for Siemens Medical Solutions. Thomas Beyer is an employee of cmi-experts.

Open Access This article is distributed under the terms of the Creative Commons Attribution Noncommercial License which permits any noncommercial use, distribution, and reproduction in any medium, provided the original author(s) and source are credited.

\section{References}

1. Czernin J, Allen-Auerbach M, Schelbert H. Improvements in cancer staging with PET/CT: literature-based evidence as of September 2006. J Nucl Med 2007;48(Suppl 1):78S-88S.

2. Kinahan $\mathrm{P}$, Hasegawa $\mathrm{B}$, Beyer T. X-ray-based attenuation correction for positron emission tomography/computed tomography scanners. Semin Nucl Med 2003;33:166-79.
3. Kinahan PE, Townsend DW, Beyer T, Sashin D. Attenuation correction for a combined 3D PET/CT scanner. Med Phys 1998;25 (10):2046-53.

4. Burger C, Goerres G, Schoenes S, Buck A, Lonn AH, Von Schulthess GK. PET attenuation coefficients from CT images: experimental evaluation of the transformation of CT into PET 511-keV attenuation coefficients. Eur J Nucl Med 2002;29:922-7.

5. Schlemmer H, Pichler B, Wienhard K, Schmand M, Nahmias C, Townsend D, et al. Simultaneous MR/PET for brain imaging: first patient scans (abstract). J Nucl Med 2007;48(Suppl 2):45P.

6. Judenhofer MS, Wehrl HF, Newport DF, Catana C, Siegel SB, Becker M, et al. Simultaneous PET-MRI: a new approach for functional and morphological imaging. Nat Med 2008;14: 459-65.

7. Catana C, Procissi D, Wu Y, Judenhofer MS, Qi J, Pichler BJ, et al. Simultaneous in vivo positron emission tomography and magnetic resonance imaging. Proc Natl Acad Sci U S A 2008;105:3705-10.

8. Zaidi H, Montandon M-L, Slosman DO. Attenuation compensation in cerebral 3D PET: effect of the attenuation map on obsolete and relative quantitation. Eur J Nucl Med Mol Imaging 2004;31:52-3.

9. Beyer T, Weigert M, Quick HH, Pietrzyk U, Vogt F, Palm C, et al. MR-based attenuation correction for torso-PET/MR imaging: pitfalls in mapping MR to CT data. Eur J Nucl Med Mol Imaging 2008;35:1142-6.

10. Shao Y, Cherry SR, Farahani K, Meadors K, Siegel S, Silverman RW, et al. Simultaneous PET and MR imaging. Phys Med Biol 1997;42:1965-70.

11. Le Goff-Rougetet R, Frouin V, Mangin J-F, Bendriem B. Segmented MR images for brain attenuation correction in PET (abstract). Proc SPIE 1994;2167:725

12. Zaidi H, Montandon M-L, Slosman DO. Magnetic resonance imaging-guided attenuation and scatter corrections in threedimensional brain positron emission tomography. Med Phys 2003;30:937-48.

13. Hofmann M, Steinke F, Aschoff P, Lichy M, Brady M, Schölkopf $\mathrm{B}$, et al. MR-based PET attenuation correction - initial results for whole body. IEEE Medical Imaging Conference, Dresden, 19-25 October 2008. M14-4.

14. El Fakhri G, Kijewski MF, Johnson KA, Syrkin G, Killiany RJ, Becker JA, et al. MRI-guided SPECT perfusion measures and volumetric MRI in prodromal Alzheimer disease. Arch Neurol 2003;60:1066-72.

15. Zaidi H, Diaz-Gomez M, Boudraa A, Slosman DO. Fuzzy clustering-based segmented attenuation correction in whole-body PET imaging. Phys Med Biol 2002;47:1143-60.

16. Dogdas B, Shattuck DW, Leahy RM. Segmentation of skull and scalp in 3-D human MRI using mathematical morphology. Hum Brain Mapp 2005;25:273-85.

17. Kops RE, Herzog H. Alternative methods for attenuation correction for PET images in MR-PET scanners. Nuclear Science Symposium Conference Record, 2007. NSS '07. IEEE. Vol. 6. p. 4327-30. doi:10.1109/NSSMIC.2007.4437073.

18. Hofmann M, Steinke F, Scheel V, Charpiat G, Farquhar J, Aschoff $\mathrm{P}$, et al. MRI-based attenuation correction for PET/MRI: a novel approach combining pattern recognition and atlas registration. $\mathrm{J}$ Nucl Med 2008;49:1875-83.

19. Friston KJ, Ashburner J, Poline JB, Frith CD, Heather JD, Frackowiak RSJ. Spatial registration and normalization of images. Hum Brain Mapp 1995;2:165-89.

20. Beyer T, Kinahan PE, Townsend DW, Sashin D. The use of X-ray CT for attenuation correction of PET data. In: Trendler RC, editor. Proceedings of the 1994 IEEE Nuclear Science Symposium and Medical Imaging Conference. Vol. 4. 1994. p. 1573-7.

21. Martínez-Möller A, Souvatzoglou M, Botnar R, Navab N, Ziegler $\mathrm{S}$, Schwaiger M, et al. An approach for MR-based attenuation 
correction for combined MR/PET: effects of ignoring bones (abstract). J Nucl Med 2007;48 (Suppl 2):156P.

22. Martínez-Möller A, Souvatzoglou M, Navab N, Schwaiger M, Nekolla S. MR-based attenuation correction for whole-body MR/ PET (abstract). J Nucl Med 2008;49(Suppl 1):65P.

23. Waldman A, Rees JH, Brock CS, Robson MD, Gatehouse PD, Bydder GM. MRI of the brain with ultra-short echo-time pulse sequences. Neuroradiology 2003;45:887-92.

24. Robson MD, Bydder GM. Clinical ultrashort echo time imaging of bone and other connective tissues. NMR Biomed 2006;19:765-80.

25. Du J, Bydder M, Takahashi AM, Chung CB. Two-dimensional ultrashort echo time imaging using a spiral trajectory. Magn Reson Imaging 2008;26:304-12.

26. Keyes JW Jr. SUV: standard uptake or silly useless value. J Nucl Med 1995;36:1836-69.

27. Pietrzyk U. Does PET/CT render software fusion obsolete? Nuklearmedizin 2005;44 (Suppl 1):S13-7.

28. Mawlawi O, Erasmus JJ, Pan T, Cody DD, Campbell R, Lonn $\mathrm{AH}$, et al. Truncation artifact on PET/CT: impact on measurements of activity concentration and assessment of a correction algorithm. AJR Am J Roentgenol 2006;186:1458-67.

29. Tsukamoto E, Ochi S. PET/CT today: system and its impact on cancer diagnosis. Ann Nucl Med 2006;20:255-67.

30. Beyer T, Bockisch A, Kühl H, Martinez MJ. Whole-Body 18F-FDG $\mathrm{PET} / \mathrm{CT}$ in the presence of truncation artifacts. J Nucl Med 2006;47:91-9.

31. Bockisch A, Beyer T, Antoch G, Freudenberg LS, Kühl H, Debatin JF, et al. Positron emission tomography/computed tomography - imaging protocols, artifacts, and pitfalls. Mol Imaging Biol 2004;6:188-99.
32. Delso G, Torres I, Bundschuh RA, Martinez-Möller A, Ziegler S, Schwaiger M, et al. Auswertung des Einflusses eines verminderten Sichtfeldes in der MR/PET Schwächungskorrektur. Nuklearmedizin 2008;2:A62-3.

33. Nehmeh S, Erdi YE, Pan T, Pevsner A, Rosenszweig KE, Yorke E, et al. Four-dimensional (4D) PET/CT imaging of the thorax. Med Phys 2004;31:3179-86.

34. Pönisch F, Richter C, Just U, Enghardt W. Attenuation correction of four dimensional (4D) PET using phase-correlated 4Dcomputed tomography. Phys Med Biol 2008;53:N259-68.

35. Leahy RM, Yan X. Incorporation of anatomical MR data for improved functional imaging with PET. Lecture Notes in Computer Science, vol. 511. Information Processing in Medical Imaging. Berlin: Springer; 1991. p. 105-20.

36. Lipinski B, Herzog H, Rota Kops E, Oberschelp W, MüllerGärtner HW. Expectation maximization reconstruction of positron emission tomography images using anatomical magnetic resonance information. IEEE Trans Med Imaging 1997; $16: 129-36$.

37. Nuyts $J$. The use of mutual information and joint entropy for anatomical priors in emission tomography. Nuclear Science Symposium Conference Record, 2007. NSS '07. IEEE. Vol. 6. p. 4149-54. doi:10.1109/NSSMIC.2007.4437034

38. Meltzer CC, Leal JP, Mayberg HS, Wagner HN Jr, Frost JJ. Correction of PET data for partial volume effects in human cerebral cortex by MR imaging. J Comput Assist Tomogr 1990; 14:561-70.

39. Meltzer CC, Kinahan PE, Breer PJ, Nichols TE, Comtat C, Cantwell MN, et al. Comparative evaluation of MR-based partialvolume correction schemes for PET. J Nucl Med 1999;40:2053-65. 